\title{
Genome-scale insights into the metabolic versatility of Limosilactobacillus reuteri
}

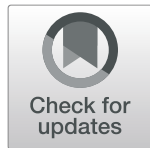

Hao Luo ${ }^{1}$, Peishun Li ${ }^{1}$, Hao Wang ${ }^{1,2,3}$, Stefan Roos ${ }^{4}$, Boyang Ji ${ }^{1}$ and Jens Nielsen ${ }^{1,5^{*}}$

\begin{abstract}
Background: Limosilactobacillus reuteri (earlier known as Lactobacillus reuteri) is a well-studied lactic acid bacterium, with some specific strains used as probiotics, that exists in different hosts such as human, pig, goat, mouse and rat, with multiple body sites such as the gastrointestinal tract, breast milk and mouth. Numerous studies have confirmed the beneficial effects of orally administered specific L. reuteri strains, such as preventing bone loss and promoting regulatory immune system development. L. reuteri ATCC PTA 6475 is a widely used strain that has been applied in the market as a probiotic due to its positive effects on the human host. Its health benefits may be due, in part, to the production of beneficial metabolites. Considering the strain-specific effects and genetic diversity of $L$. reuteri strains, we were interested to study the metabolic versatility of these strains.
\end{abstract}

Results: In this study, we aimed to systematically investigate the metabolic features and diversities of L. reuteri strains by using genome-scale metabolic models (GEMs). The GEM of L. reuteri ATCC PTA 6475 was reconstructed with a template-based method and curated manually. The final GEM iHL622 of L. reuteri ATCC PTA 6475 contains 894 reactions and 726 metabolites linked to 622 metabolic genes, which can be used to simulate growth and amino acids utilization. Furthermore, we built GEMs for the other $35 \mathrm{~L}$. reuteri strains from three types of hosts. The comparison of the L. reuteri GEMs identified potential metabolic products linked to the adaptation to the host.

Conclusions: The GEM of $L$. reuteri ATCC PTA 6475 can be used to simulate metabolic capabilities and growth. The core and pan model of $35 \mathrm{~L}$. reuteri strains shows metabolic capacity differences both between and within the host groups. The GEMs provide a reliable basis to investigate the metabolism of L. reuteri in detail and their potential benefits on the host.

Keywords: Limosilactobacillus reuteri, Lactobacillus, Probiotic, Genome-scale metabolic model, Metabolic versatility, Core metabolism, Pan metabolism

\section{Introduction}

Probiotics are "live microorganisms that, when administered in adequate amounts, confer a health benefit on the host" and many of them are applied in disease treatment and food products $[1,2]$. Some specific strains of Limosilactobacillus reuteri, previously known as Lactobacillus reuteri [3], are widely used as probiotics. $L$.

\footnotetext{
*Correspondence: nielsenj@chalmers.se

'Department of Biology and Biological Engineering, Chalmers University of Technology, SE412 96 Gothenburg, Sweden

${ }^{5}$ Biolnnovation Institute, Ole Måløes Vej 3, DK2200 Copenhagen N, Denmark Full list of author information is available at the end of the article
}

reuteri are able to colonize in a wide variety of mammals and birds affecting the hosts' health and metabolism. As a lactic acid bacterium that is generally recognized as a safe microorganism [4], some $L$. reuteri strains has been applied in a large variety of food products and food supplements throughout the world $[1,4]$, and also exploited as a potential cell factory [5]. With the shown beneficial properties of Lactobacillus/Limosilactobacillus strains, $L$. reuteri proved to have positive effects on several diseases such as improving symptoms of infantile colic, reducing diarrhea in children, preventing bone loss in the elderly and promoting regulatory immune system development

(c) The Author(s). 2021 Open Access This article is licensed under a Creative Commons Attribution 4.0 International License, which permits use, sharing, adaptation, distribution and reproduction in any medium or format, as long as you give appropriate credit to the original author(s) and the source, provide a link to the Creative Commons licence, and indicate if changes were made. The images or other third party material in this article are included in the article's Creative Commons licence, unless indicated otherwise in a credit line to the material. If material is not included in the article's Creative Commons licence and your intended use is not permitted by statutory regulation or exceeds the permitted use, you will need to obtain permission directly from the copyright holder. To view a copy of this licence, visit http://creativecommons.org/licenses/by/4.0/ The Creative Commons Public Domain Dedication waiver (http://creativecommons.org/publicdomain/zero/1.0/) applies to the data made available in this article, unless otherwise stated in a credit line to the data. 
and function [1, 6-10]. The L. reuteri ATCC PTA 6475 could prevent bone loss in a menopausal ovariectomized mouse model and were contented in chewable tablets as a dietary supplement [11-13]. Due to these advantages and possibilities, the interests of studying $L$. reuteri have significantly increased in recent years [14-16].

The benefactions in metabolism may be due, in part, to the production of metabolites such as reuterin, histamine, vitamins and exopolysaccharide [17]. For example, histamine suppresses expression of tumor necrosis factor alpha and reuterin is known as an antimicrobial compound [18]. Interestingly, L. reuteri shows strain-specific effects on human health $[17,19]$. Even among humanderived $L$. reuteri strains, the ability to reduce intestinal inflammation varies [19]. Recent studies have revealed the genetic diversity of $L$. reuteri strains [20, 21], which revealed that the diversification of $L$. reuteri strains could result from host-driven evolution, and some functional genes may be attributable to host-specific features $[20,21]$. We were therefore interested to study the metabolism of individual L. reuteri strains in detail [8].

Genome-scale metabolic models (GEMs) are useful tools in metabolic engineering that could help us to understand the metabolism and physiology of the organism [22-24]. GEMs provide a way to integrate genome sequences, experimental data, and other types of data efficiently, as a platform to connect experimental data with internal metabolic mechanisms. The GEMs of several species from family Lactobacillaceae, such as Lactobacillus plantarum [25], Lactobacillus casei [26], Lactobacillus reuteri $[5,27]$ had been reconstructed and applied for simulation related to food fermentation, probiotics, and potential cell factory.

In this study, we reconstructed a comprehensive GEM for $L$. reuteri ATCC PTA 6475, namely $i$ HL622, using a template-based method. To explore its metabolic characteristics as a probiotic strain, we simulated the growth with different carbon sources, amino acids usages and biosynthesis pathways of valuable products with experimental data. In order to explore the metabolic diversification of $L$. reuteri strains from different hosts, we further reconstructed metabolic networks for $35 \mathrm{~L}$. reuteri strains. Comparison between $L$. reuteri strains revealed potential metabolic reactions related to host adaptation.

\section{Materials and methods}

\section{Genome sequences}

One of the genome sequences of $L$. reuteri ATCC PTA 6475 we used was provided by BioGaia, and the genome annotation was performed by the Prokka software [28] and the COG database. We also used two additional genomes of this strain sequenced by the Human Microbiome Project [29], which were collected from the NCBI database with accession numbers of NZ_ACGX00000000 and GCF_000159475.2 [29]. For the core and pan -models of the $L$. reuteri species, we collected 35 strains listed in a previous study [20], which could be downloaded from NCBI (Table S1). The genome comparison was performed with BLASTP [30, 31] with following parameters: E value $<=1 \mathrm{E}-10$; bit score $>=100$; percentage of positive scoring matches $>=45 \%$. The sequences analysis was performed by the open-source package Biopython [32].

\section{Generation of $L$. reuteri GEMs}

The GEM $i$ HL622 of $L$. reuteri ATCC PTA 6475 was constructed by a template-based method with four templates, $i$ NF517 (Lactobacillus casei MG1363) [26], LbReuteri (L. reuteri JCM 1112) [5, 27], iML1515 (Escherichia coli MG1655, 33], and iBT721 (Lactobacillus plantarum WCFS1) [25]. As shown in Fig. 1a, a semi-automatic pipeline was developed for GEMs reconstruction. The $i$ NF517 was employed as the main template to build the initial draft model, and orthologs genes were identified by the best bidirectional best hits (BBHs) from BLASTP results, with the parameters: $\mathrm{E}$ value $<=1 \mathrm{E}-10$; bit score $>=100$; percentage of positive scoring matches $>=45 \%$. Then, the enzymes and associated reactions were integrated into the initial draft GEM by comparison against LbReuteri [5], $i$ ML1515 [33] and $i$ BT721 [25] one by one, this order takes into account homology and Memote scores. The exchange reactions and transport reactions were added according to the transporter annotations and corresponding medium composition. The default exchange reactions in our model corresponding a chemically defined medium with $111 \mathrm{mM}$ glucose and serial amino acids like arginine that adopted from template model of LbReuteri. More medium conditions and description can be found in references $[5,27]$. The gap-filling was performed with COBRApy [33-35] and used $i$ NF517 as a template network. The resulting GEM was manually curated using the RAVEN [36] toolbox and reactions from the MetaCyc [37] database to improve the model performance. Since $L$. reuteri ATCC PTA 6475 is a well-studied probiotic, some potentially health-related metabolites could be produced such as lactate, acetate, ethanol, 1-propanol [38], and 1,3-propanediol [39], reuterin [40] (3-hydroxypropionaldehyde), histamine, vitamin B12 [41-43] (cobalamin) and vitamin B9 [42, 44] (folate). Therefore, missing reactions involving in these pathways were introduced into the draft GEM based on references and databases. For example, production of reuterin (3-hydroxypropionaldehyde) from glycerol is not annotated automatically but introduced manually.

After functional curation, the mass balance, charge balance and information annotation of the GEM were checked. To make the GEM recognized in other namespaces and connected with other databases, we generated annotations of EC number and the links to 


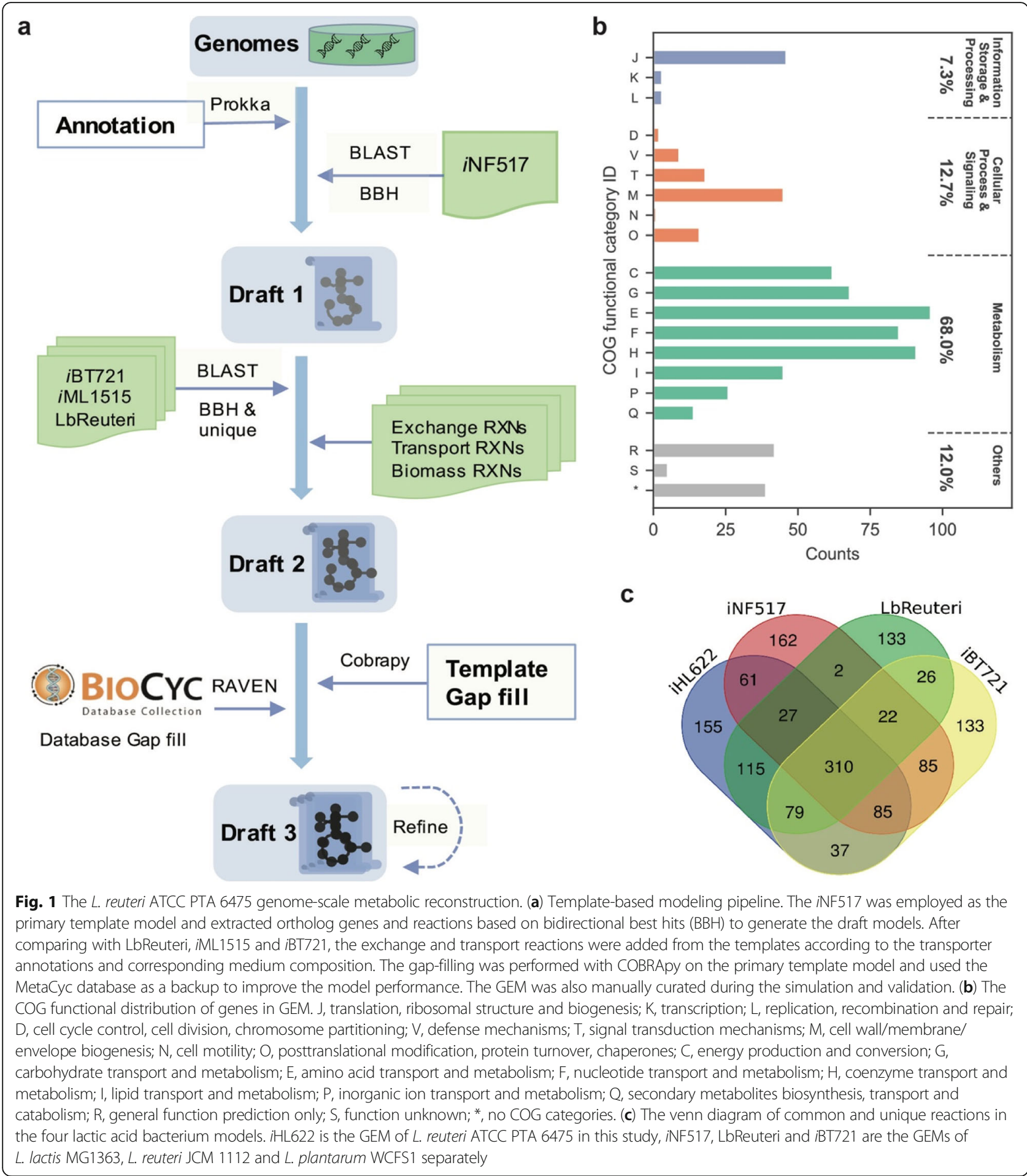

databases such as MetaCyc, KEGG and MetaNetX. Furthermore, MEMOTE [45] was applied to assess the GEM quality. With the proteome sequences of 35 strains, we performed the GEMs reconstruction for each strain as previously described [20].

The biomass reaction was adopted from the template models. The DNA coefficients were generated by the
BOFdat toolbox [46]. The fraction weight of DNA was set to $0.031 \mathrm{~g}$ of $1 \mathrm{~g}$ biomass, and the detail coefficients of each nucleotide were set according to the DNA sequence GC content. The biomass content and composition of protein and lipid fractions were recalculated based on the LbReuteri model [5]. The code and model files could be found at a public GitHub repository 
(https://github.com/SysBioChalmers/Lactobacillus_ reuteri_MM41A_GEM).

\section{Flux balance analysis}

Growth capabilities in different mediums and essential amino acids validation were tested by flux balance analysis (FBA) [47]. We set the constraints of exchange reactions of medium and amino acids in the model with experimental substrate uptake rates and set the biomass reaction as the objective function to test the growth capability. During the testing of essential amino acids, Dglucose was selected as the sole carbon source and the uptake rate was set as $25 \mathrm{mmol} / \mathrm{g} \mathrm{DW} / \mathrm{h}$ from reference. When we tested the essential amino acids for growth, we set the uptake rate of 20 amino acids as $0 \mathrm{mmol} / \mathrm{g}$ [CDW] / h respectively. Growth rates under $1 \mathrm{E}-10$ or infeasible results were considered as no growth. To test the synthesis capacities of products, we set secretion reaction of corresponding products as the object function to perform FBA. Secretory rates above zero pointed to that the model has corresponding synthesis capacity. The simulations were carried out in Python 3.7.9 with the COBRApy [33] 0.20.0 package and CPLEX optimizer 12.5.1 from IBM.

\section{Results and discussion}

\section{The characteristics of $L$. reuteri ATCC PTA 6475 GEM}

Three L. reuteri ATCC PTA 6475 genome sequences from different sources were collected. Two of them had been sequenced by the Human Microbiome Project [29] and the third one had been sequenced by BioGaia. Genome annotation of L. reuteri ATCC PTA 6475 from BioGaia yielded 2019 protein-encoding genes, 71 tRNA and 18 rRNA genes.
Functional analysis based on clusters of orthologous groups (COG) classification showed that $80.5 \%$ of protein-encoding genes were mapped into COG categories. As shown in Fig. S1, 28.6\% genes were related with metabolism and 26.3\% genes associated with cellular process and signaling. The top three most abundant functional categories were 'Mobilome: prophages, transposons' $(\mathrm{X})$, 'Translation, ribosomal structure and biogenesis'(J) and 'Amino acid transport and metabolism'(E). Comparative genomic analysis shows that 1852 genes (93.17\% on average) are shared between genomes from the three sources (Fig. S1b), while 102 protein-encoding genes were specific for strain from BioGaia. These three genome sequences got same complete value of $98.4 \%$ by BUSCO [48] analysis and the $L$. reuteri ATCC PTA 6475 genome sequence from BioGaia with only one contig was employed to reconstruct the GEM.

As shown in Fig. 1a, the GEM $i$ HL662 was reconstructed by a template-based method. The initial draft model including 383 reactions and 465 metabolites was developed using $i$ NF517 as template based on 763 BBHs. The metabolic genes and associated reactions mapping to the other three template models were also integrated into the draft model based on BBHs. The exchange reactions and transport reactions were added to enable nutrient uptake and by-product secretion, and gap-filling was performed to enable growth and by-product production. Furthermore, manual curations were conducted to remove potential errors in reactions or metabolites. Altogether, the final model $i$ HL622 was obtained including 869 reactions and 713 metabolites with intracellular and extracellular components (Table 1), which is associated with 623 genes (30.8\% of the genome) and 584 of them with COG categories (Fig. 1b). Compared with

Table 1 Model characteristics of iHL622 and comparison with template GEMs

\begin{tabular}{|c|c|c|c|c|c|}
\hline Model & iHL622 & iNF517 & LbReuteri & iBT721 & iML1515 \\
\hline Organism & L. reuteri ATCC PTA 6475 & L.lactis MG1363 & $\begin{array}{l}\text { L. reuteri } \\
\text { JCM } 1112\end{array}$ & L. plantarum WCFS1 & $\begin{array}{l}\text { E. coli } \\
\text { MG1655 }\end{array}$ \\
\hline Genes & 2019 & 2339 & 1943 & 3063 & 4243 \\
\hline Included & $622(31 \%)$ & $516(22 \%)$ & $530(27 \%)$ & 724 (24\%) & $1516(36 \%)$ \\
\hline Reactions & 869 & 754 & 714 & 778 & 2712 \\
\hline Common with iHL622 & 869 & 483 & 531 & 392 & 509 \\
\hline With GPR ${ }^{a}$ & 709 (82\%) & $541(72 \%)$ & $606(85 \%)$ & $528(68 \%)$ & $2266(86 \%)$ \\
\hline Internal & 644 & 530 & 507 & 538 & 1548 \\
\hline Transport & 122 & 119 & 123 & 127 & 833 \\
\hline Exchange & 103 & 105 & 84 & 113 & 331 \\
\hline Metabolites & 713 & 650 & 660 & 662 & 1877 \\
\hline Unique & 605 & 545 & 561 & 549 & 1071 \\
\hline Biomass consistency & 1.00 & 0.83 & $-b$ & $-b$ & 1.00 \\
\hline MEMOTE Score & $80 \%$ & $60 \%$ & $57 \%$ & $38 \%$ & $68 \%$ \\
\hline
\end{tabular}

a Gene-Protein-Reaction Associations

b Not applicable 
other published GEMs, there are 392 to 531 common reactions and 155 unique reactions in $i$ HL622 (Table 1 and Fig. 1c). In addition, $i$ HL622 included 31\% more genes than the other three lactic acid bacterium templates models and there are $82 \%$ reactions in $i$ HL622 associated with genes. MEMOTE analysis also showed the highest quality of $i$ HL622 comparison against other GEMs.

\section{Prediction of L. reuteri ATCC PTA 6475 growth with different substrates}

$i$ HL622 was employed to simulate growth of $L$. reuteri ATCC PTA 6475 under different growth conditions (Fig. 2). A previous study revealed that $L$. reuteri JCM1112, a highly similar strain of L. reuteri ATCC PTA $6475[49,50]$, grows faster with glycerol supplied and predominantly using the phosphoketolase (PK) pathway [5]. Therefore, $i$ HL622 was used to simulate the growth capability with only glucose and with both glucose and glycerol by constraining the carbon sources uptake rates, and the exchange fluxes of other extracellular metabolites. Some studies have described the importance of $L$. reuteri's glycolytic pathway and we also found that it could significantly affect the growth rate, so we added constrains of maximum flux of Embden-Meyerhof-Parnas (EMP) pathway to curate relevant pathways $[5,51]$. Since both EMP and PK pathways exist in L. reuteri, the PK pathway should be dominant pathway, even the EMP pathway could provide more energy yield than the PK pathway [5, 51]. When reducing the EMP pathway flux with constraints on phosphofructokinase (PFK) and fructose-1,6biphosphate aldolase (FBA) reactions [5], the growth rate was reduced significantly and close to the experimental data $[5,51,52]$, which coincides with reports that the PK pathway shared the main carbon flux $[5,51]$. In addition, the constraints of amino acids uptake rates and secretion

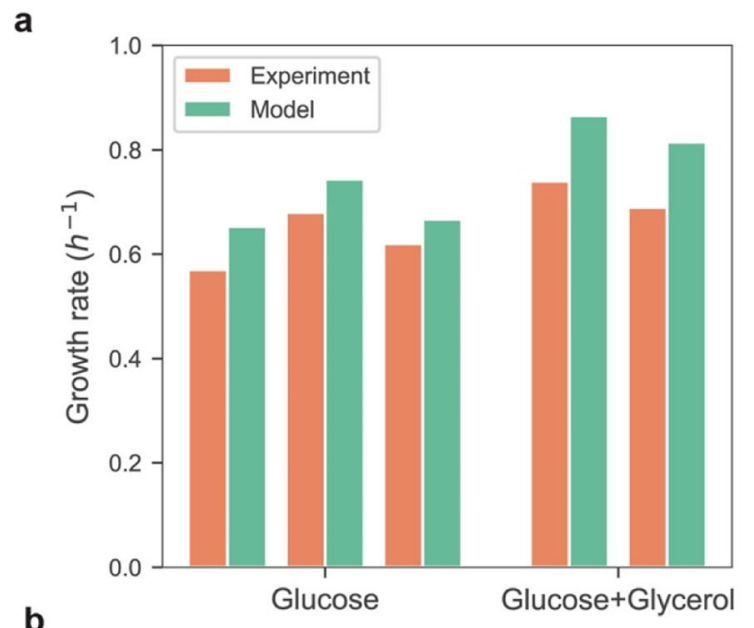

C

b
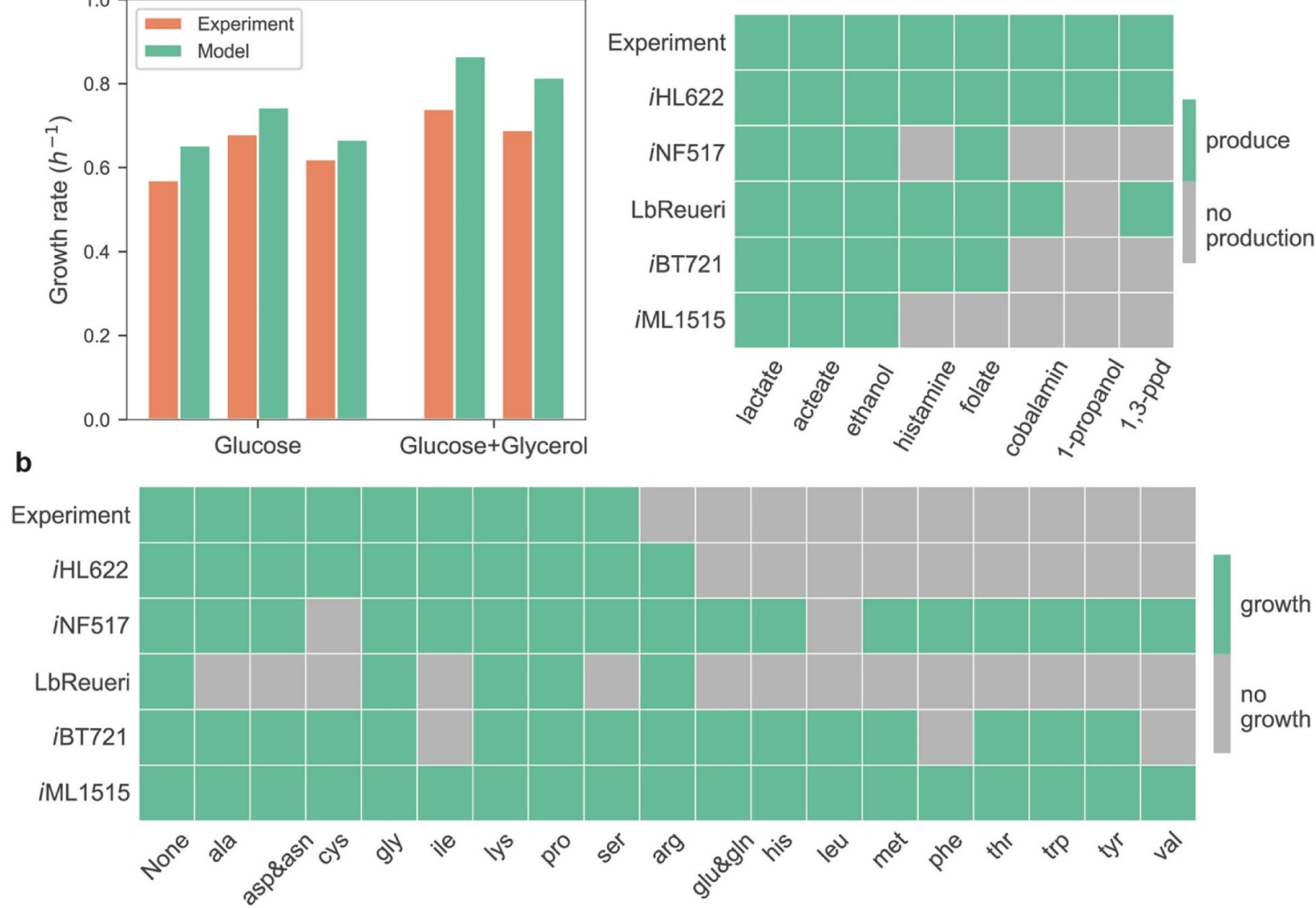

Fig. 2 The predictions based on the iHL622 GEM. (a) Experimental and predicted growth rates. The experimental data for each dataset are shown in orange, and the prediction showing in green. (b) Growth capability under amino acid omitted medium. Experimental data are showed in the first row, while the predictions of single amino acid omission are shown in the rest rows. The growth showing in green and no growth showing in grey. (c) The predictions of representative metabolites. Eight products (lactate, acetate, ethanol, histamine, folate, cobalamin, 1-propanol and 1,3-propanediol) were predicted. Experimental data are showed in the first row, while the modeling results are shown in the rest rows. The produce showing in green and no productions showing in grey 
rates of lactate and acetate were also added based on the experimental data. Altogether, the predicted specific growth rates are consistent with experimental observations [5]. The experimental growth rates are $0.751 \pm 0.03$ $\mathrm{h}^{-1}$ with glycerol supplementation and $0.623 \pm 0.04 \mathrm{~h}^{-1}$ without glycerol, both are close to the values predicted by the model.

Moreover, $i$ HL622 was used to predict the growth capability of $L$. reuteri ATCC PTA 6475 using amino acid as nitrogen sources (Fig. 2b). A previous study showed that eight non-essential amino acids (alanine, aspartate, cysteine, glycine, isoleucine, lysine, proline, serine) could be omitted from the growth medium and affected the growth rate and vitamin B12 production to different degrees [41]. The study also mentioned that the omission of serine reduced the specific growth rate about $80 \%$ whereas omission of other amino acids only caused $13 \%$ reduction on average [41]. The qualitative predictions of single amino acid omission predictions are matching literature results except for arginine [41]. Since the uptake rate of amino acids were not mentioned in the reference, quantitative prediction was not performed. The mismatch between arginine predictions and experimental data may be due to the inherited characteristics from template GEMs and insufficient annotation of related enzymes.

Previous studies show that $L$. reuteri strains have the capacities to synthesize lactate, acetate, ethanol [53], histamine, folate [42], cobalamin [41-43], 1-propanol [5456] and 1,3-propanediol [57]), which may be linked to the probiotic effects of L. reuteri. As shown in Fig. 2c, the biosynthesis capacities of $i$ HL622 for these products were explored, and production of all these metabolites can be predicted correctly by $i$ HL622.

\section{The metabolic features of $L$. reuteri ATCC PTA 6475}

As a probiotic strain, $L$. reuteri ATCC PTA 6475 may affect host metabolism directly through secretion of metabolites that are influencing human cells. In order to investigate the health-promoting properties and metabolic features of $L$. reuteri ATCC PTA 6475, the main metabolic pathways were investigated tracked (Fig. 3). As mentioned before, carbohydrate metabolism mainly uses the PK pathway to produce lactate, acetate and ethanol, not EMP or EMP extensions even though they all appear in our model $[5,51,52]$. The PK pathway regulated by ribulose epimerase (MBLCLPDI_01299) and phosphoketolase (MBLCLPDI_01842) in the model. In food fermentation, lactate is usually the most important endproduct of fermentation by lactobacilli, acetate and ethanol are main by-products, but the composition of the final end-products change dependent on growth conditions [4]. Due to its potential use as a biofuel, biosynthesis of 1-propanol has been extensively studied [54-
56], and this metabolite can be produced from both glucose or glycerol. Here we focused on histamine and 3HPA (reuterin), two potential beneficial metabolites synthesis genes and pathways. The histamine is a potential immunomodulatory factor that can modulate host mucosal immunity and suppresses pro-inflammatory tumor necrosis factor alpha production [18]. L. reuteri ATCC PTA 6475 have the histamine biosynthesis pathway and transporters that can convert L-histidine to histamine via histidine/histamine antiporter (hdcP, MBLCLPDI 01994), histidine decarboxylase pyruvoyl type A (hdcA, MBLCLPDI_01992), and hdcB (hdcB, MBLCLPDI_ 01991) [58]. The predicted histidine decarboxylase showed 95\% identities against the histidine decarboxylase from conformed $L$. reuteri strains. 3Hydroxypropionaldehyde (3-HPA) is the main component of reuterin that acts as a broad-spectrum antimicrobial substance and is an intermediate of the 1,3propanediol synthesis pathway [57]. The 3-HPA production needs a one-step reaction from glycerol by the B12dependent glycerol/diol dehydratase (PduC, PduD, PduE) [57, 59], which are encoded by the genes MBLCLPDI_01903, MBLCLPDI_01902 and MBLCLPDI_01901.

\section{Core and pan metabolism of $L$. reuteri}

Limosilactobacillus species have been isolated from a wide range of sources. Recent genome sequencing of Limosilactobacillus species has provided basis to explore the metabolic diversity of Limosilactobacillus at the genome level $[20,21]$. Some studies report that $L$. reuteri species from different ecological origins are closely associated with their living environment and genomic diversity $[20,21]$. They also found some functional genes attributable to the host such as genes encoding cell surface proteins and active carbohydrate enzymes [20]. Here we analyzed the $L$. reuteri metabolism by metabolic modeling. The genome sequences of $35 \mathrm{~L}$. reuteri strains were collected and used for GEMs reconstruction. These 35 strains can be classified into three distinct groups based on their corresponding host including herbivore, omnivore, and sourdough, with a distribution of 16,15 , and four strains into the three groups respectively. The genome size and GEMs characteristics are shown in Fig. 4a, with a genome size of $2058.3 \pm 222.9$ CDS and GEMs of $919.8 \pm 35.0$ reactions and $811.0 \pm 25.7$ metabolites linked to $567.1 \pm 35.6$ encoding genes. Here we found that the GEMs size is weakly correlated with genome size, the genome size is sorted in descending order (Fig. 4a, right) while none of the model characteristics correspond to this order (Fig. 4a, left).

Moreover, group-wised core and pan metabolic models were reconstructed. In the herbivore group, the GEMs have $929.2 \pm 26.1$ reactions and $818.8 \pm 17.3$ 


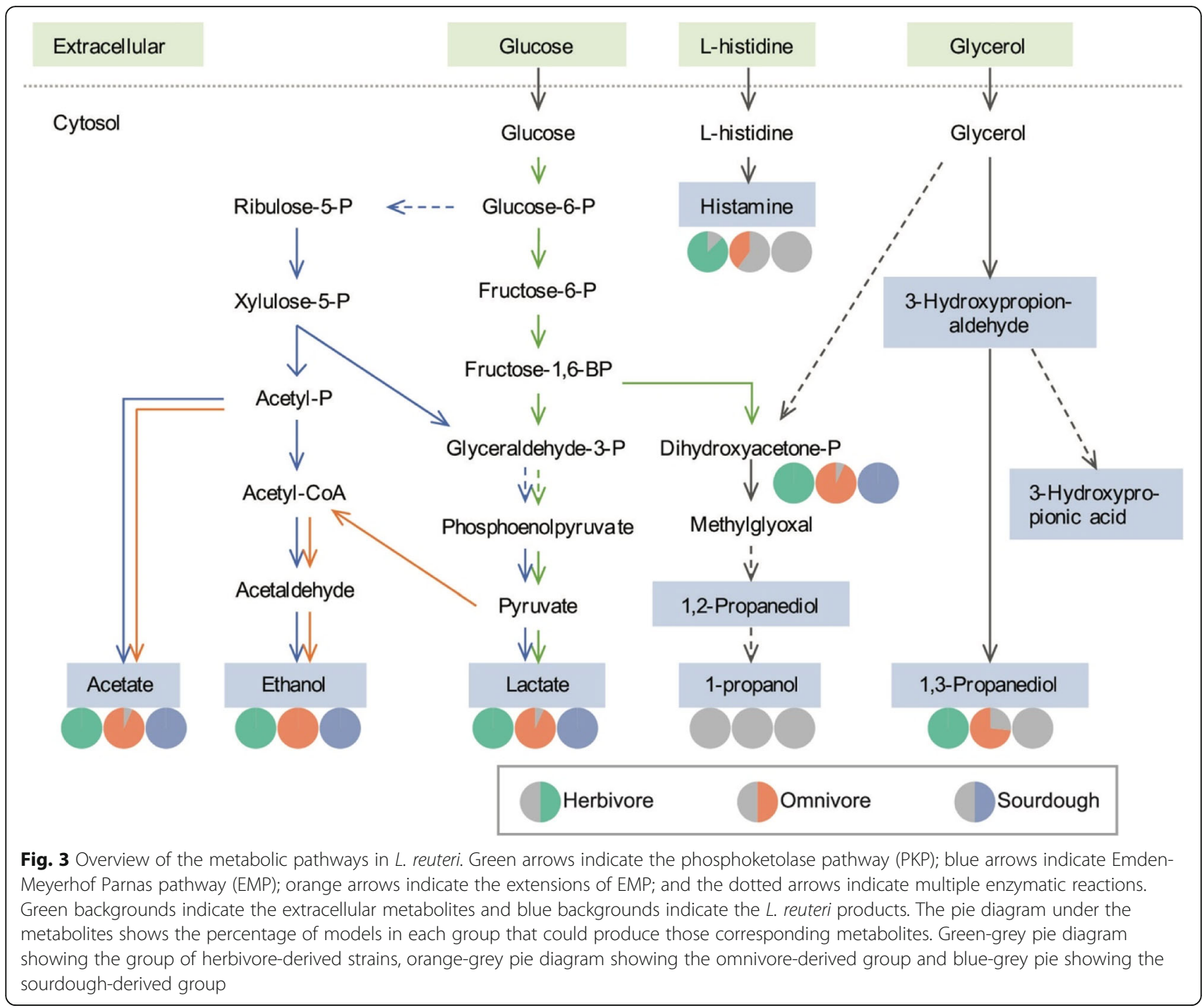

metabolites associated with $579.4 \pm 30.6$ genes. In the omnivore group, there are $924.5 \pm 30.2$ reactions and $812.8 \pm 27.4$ metabolites associated with $571.8 \pm 23.0$ genes. While in the sourdough group, there are 864.2 \pm 39.0 reactions and $773.0 \pm 14.3$ metabolites associated with $500.5 \pm 19.4$ genes. The herbivore core metabolic model included 847 reactions and 771 metabolites, corresponding to 85.9 and $90.0 \%$ of the pan model. The omnivore core metabolic model included 796 reactions and 715 metabolites, corresponding to 80.73 and $83.82 \%$ of the pan model. And the sourdough core metabolic model included 752 reactions and 726 metabolites, corresponding to 81.91 and $91.20 \%$ of pan model.

Previous comparative genomic analysis showed that there are host-specific genes in different groups ([20] and Fig. 4b). However, slight differences in reactions and metabolites were observed between these strains and groups (Fig. 4c, d). In this comparison, only appearing in one group is considered specific, appearing in all groups is considered common and appearing in two (between one and all) groups is considered dispensable. As shown in Fig. 4b, the specific and common genes are 20.7 and $50.4 \%$ respectively. In our models (Fig. 4c, d., there are $7.8 \%$ specific reactions and $5.5 \%$ specific metabolites correspondingly $74.8 \%$ common reactions and $83.7 \%$ common metabolites. Here we noticed that the percentage of specific genes is more than specific model reactions and metabolites, while the common percentage is opposite, low correlation suggests that the many of the differences in the genome are not inherited to GEMs. Finally, we obtained core and pan models of the 35 strains, containing 671 reactions and 666 metabolites in the core model, while there are 1010 reactions and 870 metabolites in the pan model.

We compared the synthesis capacities of products between the three groups and the results are shown in Fig. 3. Most of the strains have similar carbohydrate metabolism pathways and have the capacity to produce 

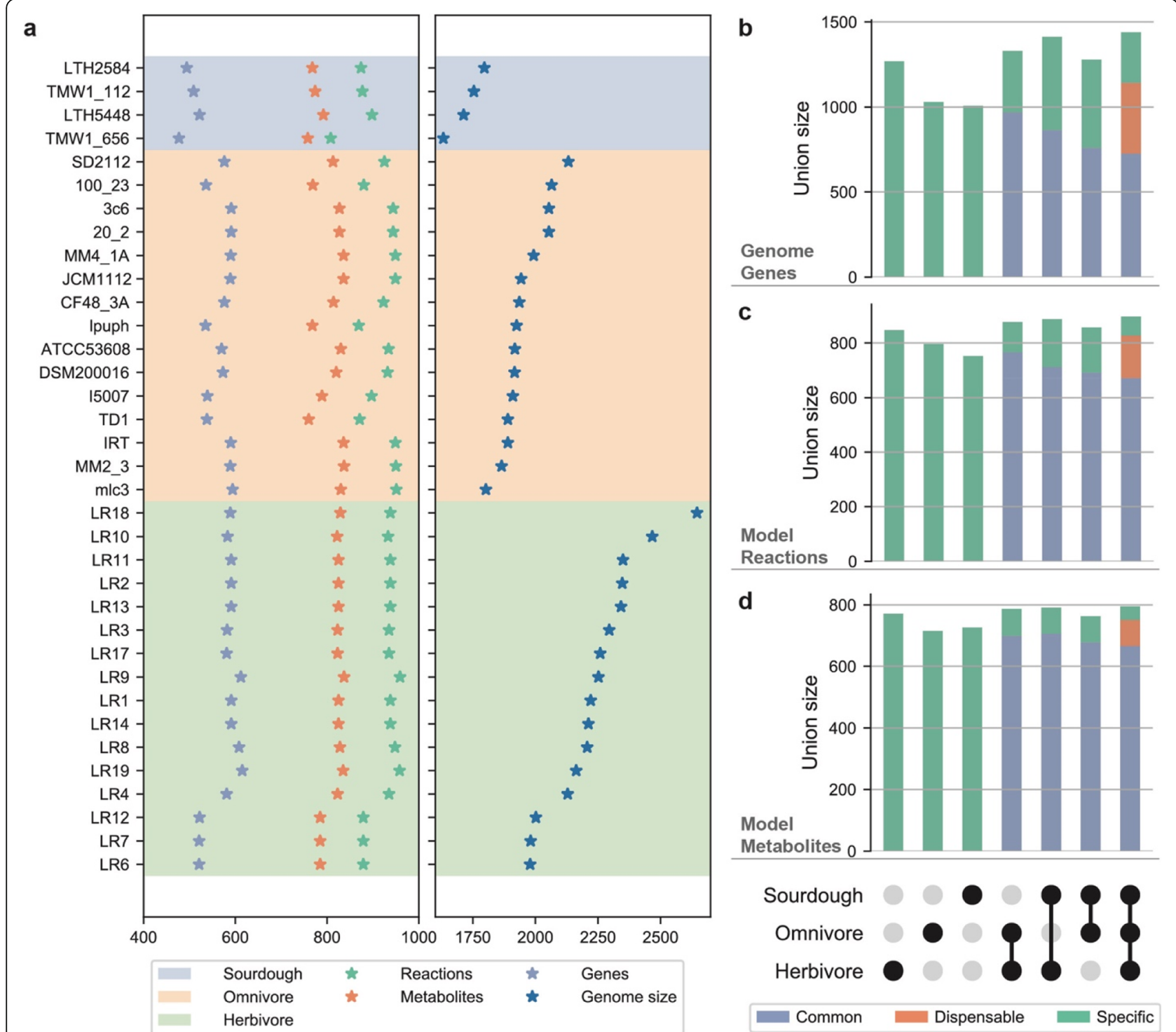

Fig. 4 Characteristics of core- and pan-GEMs of $35 \mathrm{~L}$. reuteri strains from different hosts. (a) Genome size shown in blue on the right, GEMs characteristics shown on the left. Green asterisks indicate the number of reactions, orange asterisks indicate the number of metabolites, and light blue asterisks indicate the number of genes in each GEM. These GEMs are grouped by host: herbivore, omnivore, and sourdough. The strains list in the $y$-axis are sorted in descending order by genome size in each group. (b,c, d) Upset plot of genes, reactions and metabolites between the pan-models of three groups. The total height of the bar indicates the union size of the corresponding group in the horizontal coordinate. In the final plotted bar, only appearing in one group is considered specific, appearing in all groups is considered common and appearing in two (between one and all) groups is considered dispensable. The common, dispensable and specific size from all combinations are shown

acetate, ethanol and lactate. While for strain-dependent products like histamine and 1,3-propanediol, the synthesis pathways have differences both in and between groups. We find that the herbivore-derived L. reuteri maybe have the most completed metabolic pathways related to histamine and 1,3-propanediol because most models in herbivores could produce them (Fig. 3). However, all the GEMs in the sourdough group cannot produce histamine and 1,3-propanediol, this suggests that they may have less beneficial effects on their host. The omnivore group has the most differences within the group, i.e. there are 42.9 and $78.6 \%$ have the capacity to produce histamine and 1,3-propanediol separately. And the methylglyoxal synthase ( $m g s A)$ gene was missing in all GEMs of 35 strains, which explains why 1-propanol was not produced.

From the comparison of model characteristics and synthesis capacities, we found that the metabolism of the three groups of models is very similar, after all, there are more than $95 \%$ common reactions. However, the 
differences cannot be ignored, especially the ability to provide potentially beneficial metabolites. For instance, herbivore-derived $L$. reuteri may have some advantages in producing histamine and 1,3-propanediol, which provided the potential to be explored as a probiotic.

\section{Conclusions/ discussion}

Here we reconstructed a GEM of $L$. reuteri ATCC PTA 6475 that can be used to simulate the metabolic capabilities and growth rates under different mediums. Most of GEM predictions were matched with experimental data except for the essential of arginine. Furthermore, coreand pan- GEMs of $35 \mathrm{~L}$. reuteri strains were reconstructed and based on these we identified different synthesis capacities of histamine and 1,3-propanediol among these strains. These metabolic differences demonstrate some of the advantages of herbivore-derived $L$. reuteri which could provide potential assistance in the study of strain specificity and the exploration of future industrial strains. All the GEMs of L.reuteri provide a reliable basis to investigate the metabolism of $L$. reuteri in detail and their potential benefit on host health.

\section{Abbreviations}

GEM: Genome-scale metabolic model; FBA: Flux balance analysis

\section{Supplementary Information}

The online version contains supplementary material available at https://doi. org/10.1186/s12896-021-00702-w.

Additional file 1. Model file of $i \mathrm{HL} 622$ and 35 pan-GEMs in SBML format.

Additional file 2: Fig. S1. Comparison of L. reuteri ATCC PTA 6475 sequences and COG functional distribution.

Additional file 3: Table S1. Information of $35 \mathrm{~L}$. reuteri strains.

\section{Acknowledgements}

Not applicable.

\section{Authors' contributions}

JN conceived and designed the study. SR provided genome sequences, $\mathrm{HL}$, $\mathrm{HW}$ and $\mathrm{BJ}$ performed model reconstruction and analysis, HL, PL SR, and BJ contributed to data analyses and visualization. HL wrote the first draft. PL, HW, SR, BJ and JN revised the manuscript. All authors critically reviewed and approved the manuscript.

\section{Funding}

This project was funded by Knut and Alice Wallenberg Foundation and BioGaia AB. Open Access funding provided by Chalmers University of Technology.

\section{Availability of data and materials}

All data generated or analyzed during this study are included in this published article and its supplementary information files.

\section{Declarations}

Ethics approval and consent to participate Not applicable.

\section{Consent for publication}

Not Applicable.

\section{Competing interests}

This project was partly funded by BioGaia AB.

\section{Author details}

${ }^{1}$ Department of Biology and Biological Engineering, Chalmers University of Technology, SE412 96 Gothenburg, Sweden. ${ }^{2}$ Wallenberg Centre for Molecular and Translational Medicine, University of Gothenburg, SE405 30 Gothenburg, Sweden. ${ }^{3}$ National Bioinformatics Infrastructure Sweden, Science for Life Laboratory, Chalmers University of Technology, SE412 96 Gothenburg, Sweden. ${ }^{4}$ Department of Molecular Sciences, Uppsala BioCenter, Swedish University of Agricultural Sciences, SE750 07 Uppsala, Sweden. ${ }^{5}$ Biolnnovation Institute, Ole Måløes Vej 3, DK2200 Copenhagen N, Denmark.

Received: 17 March 2021 Accepted: 22 June 2021

Published online: 30 July 2021

\section{References}

1. Mu Q, Tavella VJ, Luo XM. Role of Lactobacillus reuteri in human health and diseases. Front Microbiol. 2018:9 APR:1-17.

2. Chau K, Lau E, Greenberg S, Jacobson S, Yazdani-Brojeni P, Verma N, et al. Probiotics for infantile colic: A randomized, double-blind, placebo-controlled trial investigating Lactobacillus reuteri DSM 17938. J Pediatr. 2015;166:74-78. e1. https://doi.org/10.1016/j.jpeds.2014.09.020.

3. Zheng J, Wittouck S, Salvetti E, Franz CMAP, Harris HMB, Mattarelli P, et al. A taxonomic note on the genus Lactobacillus: description of 23 novel genera, emended description of the genus Lactobacillus beijerinck 1901, and union of Lactobacillaceae and Leuconostocaceae. Int J Syst Evol Microbiol. 2020; 70(4):2782-858. https://doi.org/10.1099/ijsem.0.004107.

4. Vankerckhoven V, Huys G, Vancanneyt M, Vael C, Klare I, Romond MB, et al. Biosafety assessment of probiotics used for human consumption: recommendations from the EU-PROSAFE project. Trends Food Sci Technol. 2008;19(2):102-14. https://doi.org/10.1016/j.tifs.2007.07.013.

5. Kristjansdottir T, Bosma EF, Branco dos Santos F, Özdemir E, Herrgård MJ, França $\mathrm{L}$, et al. A metabolic reconstruction of Lactobacillus reuteri JCM 1112 and analysis of its potential as a cell factory. Microb Cell Factories. 2019; 18(1):186. https://doi.org/10.1186/s12934-019-1229-3.

6. Giraffa G, Chanishvili N, Widyastuti Y. Importance of lactobacilli in food and feed biotechnology. Res Microbiol. 2010;161(6):480-7. https://doi.org/10.101 6/j.resmic.2010.03.001.

7. Schepper JD, Collins FL, Rios-Arce ND, Raehtz S, Schaefer L, Gardinier JD, et al. Probiotic Lactobacillus reuteri prevents Postantibiotic bone loss by reducing intestinal Dysbiosis and preventing barrier disruption. J Bone Miner Res. 2019;34(4):681-98. https://doi.org/10.1002/jbmr.3635.

8. Urbańska M, Gieruszczak-Białek D, Szajewska H. Systematic review with meta-analysis: Lactobacillus reuteri DSM 17938 for diarrhoeal diseases in children. Aliment Pharmacol Ther. 2016;43(10):1025-34. https://doi.org/1 0.1111/apt.13590.

9. Savino F, Cordisco L, Tarasco V, Palumeri E, Calabrese R, Oggero R, et al. Lactobacillus reuteri DSM 17938 in infantile colic: a randomized, doubleblind, placebo-controlled trial. Pediatrics. 2010;126(3):e526-33. https://doi. org/10.1542/peds.2010-0433.

10. Savino F, Pelle E, Palumeri E, Oggero R, Miniero R. Lactobacillus reuteri (American type culture collection strain 55730) versus simethicone in the treatment of infantile colic: a prospective randomized study. Pediatrics. 2007;119(1):e124-30. https://doi.org/10.1542/peds.2006-1222.

11. Britton RA, Irwin R, Quach D, Schaefer L, Zhang J, Lee T, et al. Probiotic L. reuteri treatment prevents bone loss in a menopausal Ovariectomized mouse model. J Cell Physiol. 2014;229(11):1822-30. https://doi.org/10.1002/ jcp.24636.

12. Dore MP, Bibbò S, Pes GM, Francavilla R, Graham DY. Role of probiotics in helicobacter pylori eradication: lessons from a study of Lactobacillus reuteri strains DSM 17938 and ATCC PTA 6475 (Gastrus ${ }^{\oplus}$ ) and a proton-pump inhibitor. Can J Infect Dis Med Microbiol. 2019;2019:1-8. https://doi.org/1 0.1155/2019/3409820.

13. Nilsson AG, Sundh D, Bäckhed F, Lorentzon M. Lactobacillus reuteri reduces bone loss in older women with low bone mineral density: a randomized, placebo-controlled, double-blind, clinical trial. J Intern Med. 2018;284(3):30717. https://doi.org/10.1111/joim.12805.

14. Wu H, Xie S, Miao J, Li Y, Wang Z, Wang M, et al. Lactobacillus reuteri maintains intestinal epithelial regeneration and repairs damaged intestinal 
mucosa. Gut Microbes. 2020;11(4):997-1014. https://doi.org/10.1080/194 90976.2020.1734423.

15. Wang $M$, Wu $H$, Lu L, Jiang L, Yu Q. Lactobacillus reuteri promotes intestinal development and regulates mucosal immune function in newborn piglets. Front Vet Sci. 2020;7:42. https://doi.org/10.3389/fvets.2 020.00042.

16. Alayande KA, Aiyegoro OA, Nengwekhulu TM, Katata-Seru L, Ateba CN. Integrated genome-based probiotic relevance and safety evaluation of Lactobacillus reuteri PNW1. PLoS One. 2020;15(7):e0235873. https://doi.org/1 0.1371/journal.pone.0235873.

17. Morita H, Hidehiro TOH, Fukuda S, Horikawa H, Oshima K, Suzuki T, et al. Comparative genome analysis of Lactobacillus renteri and Lactobacillus fermentum reveal a genomic island for reuterin and cobalamin production. DNA Res. 2008;15(3):151-61. https://doi.org/10.1 093/dnares/dsn009.

18. Greifová G, Májeková H, Greif G, Body P, Greifová M, Dubničková M. Analysis of antimicrobial and immunomodulatory substances produced by heterofermentative Lactobacillus reuteri. Folia Microbiol. 2017;62:515-24. https://doi.org/10.1007/s12223-017-0524-9.

19. Liu Y, Fatheree NY, Mangalat N, Rhoads JM. Human-derived probiotic Lactobacillus reuteri strains differentially reduce intestinal inflammation. Am J Physiol Gastrointest Liver Physiol. 2010;299(5):1087-96. https://doi.org/1 0.1152/ajpgi.00124.2010.

20. Yu J, Zhao J, Song Y, Zhang J, Yu Z, Zhang H, et al. Comparative genomics of the herbivore gut symbiont Lactobacillus reuteri reveals genetic diversity and lifestyle adaptation. Front Microbiol. 2018;9:1151. https://doi.org/10.33 89/fmicb.2018.01151.

21. Oh PL, Benson AK, Peterson DA, Patil PB, Moriyama EN, Roos S, et al. Diversification of the gut symbiont Lactobacillus reuteri as a result of host-driven evolution. ISME J. 2010;4(3):377-87. https://doi.org/10.1038/ismej.2009.123.

22. Cook DJ, Nielsen J. Genome-scale metabolic models applied to human health and disease. Wiley Interdiscip Rev Syst Biol Med. 2017;9(6). https:// doi.org/10.1002/wsbm.1393.

23. Sánchez BJ, Zhang C, Nilsson A, Lahtvee P, Kerkhoven EJ, Nielsen J. Improving the phenotype predictions of a yeast genome-scale metabolic model by incorporating enzymatic constraints. Mol Syst Biol. 2017;13:935. https://doi.org/10.15252/msb.20167411.

24. Åkesson M, Förster J, Nielsen J. Integration of gene expression data into genome-scale metabolic models. Metab Eng. 2004;6(4):285-93. https://doi. org/10.1016/j.ymben.2003.12.002.

25. Teusink B, Wiersma A, Molenaar D, Francke C, De Vos WM, Siezen RJ, et al. Analysis of growth of Lactobacillus plantarum WCFS1 on a complex medium using a genome-scale metabolic model. J Biol Chem. 2006;281(52): 40041-8. https://doi.org/10.1074/jbc.M606263200.

26. Ye C, Xu N, Chen H, Chen YQ, Chen W, Liu L. Reconstruction and analysis of a genome-scale metabolic model of Lactobacillus casei LC2W. BMC Syst Biol. 2015;9:140-7.

27. Vinay-Lara E, Hamilton JJ, Stahl B, Broadbent JR, Reed JL, Steele JL. Genome -scale reconstruction of metabolic networks of lactobacillus casei ATCC 334 and 12A. PLoS One. 2014;9(11):e110785. https://doi.org/10.1371/journal.pone.0110785.

28. Seemann T. Genome analysis Prokka: rapid prokaryotic genome annotation. Bioinformatics. 2014;30:2068-9. https://doi.org/10.1093/bioinformatics/btu153.

29. Turnbaugh PJ, Ley RE, Hamady M, Fraser-Liggett CM, Knight R, Gordon Jl. The human microbiome project. Nature. 2007;449(7164):804-10. https://doi. org/10.1038/nature06244.

30. Henikoff S, Henikoff JG. Amino acid substitution matrices from protein blocks. Proc Natl Acad Sci U S A. 1992;89(22):10915-9. https://doi.org/10.1 073/pnas.89.22.10915.

31. McGinnis S, Madden TL. BLAST: At the core of a powerful and diverse set of sequence analysis tools. Nucleic Acids Res. 2004;32(WEB SERVER):W20-5. https://doi.org/10.1093/nar/gkh435.

32. Cock PJA, Antao T, Chang JT, Chapman BA, Cox CJ, Dalke A, et al. Biopython: freely available Python tools for computational molecular biology and bioinformatics. Bioinformatics. 2009;25(11):1422-3. https://doi. org/10.1093/bioinformatics/btp163.

33. Monk JM, Lloyd CJ, Brunk E, Mih N, Sastry A, King Z, et al. iML1515, a knowledgebase that computes Escherichia coli traits. Nat Biotechnol. 2017; 35(10):904-8. https://doi.org/10.1038/nbt.3956.

34. Kumar VS, Maranas CD. GrowMatch: an automated method for reconciling in silico/in vivo growth predictions. PLoS Comput Biol. 2009;5(3):e1000308. https://doi.org/10.1371/journal.pcbi.1000308.
35. Reed JL, Patel TR, Chen KH, Joyce AR, Applebee MK, Herring CD, et al. Systems approach to refining genome annotation. Proc Natl Acad Sci U S A. 2006;103(46):17480-4. https://doi.org/10.1073/pnas.0603364103.

36. Wang H, Marcišauskas S, Sánchez BJ, Domenzain I, Hermansson D, Agren R, et al. RAVEN 2.0: a versatile toolbox for metabolic network reconstruction and a case study on Streptomyces coelicolor. PLoS Comput Biol. 2018; 14(10):e1006541. https://doi.org/10.1371/journal.pcbi.1006541.

37. Caspi R, Billington R, Fulcher CA, Keseler IM, Kothari A, Krummenacker M, et al. The MetaCyc database of metabolic pathways and enzymes. Nucleic Acids Res. 2018;46(D1):D633-9. https://doi.org/10.1093/nar/gkx935.

38. Chen $\mathrm{G}$, Chen J. A novel cell modification method used in biotransformation of glycerol to 3-HPA by Lactobacillus reuteri. Appl Microbiol Biotechnol. 2013;97(10):4325-32. https://doi.org/10.1007/s00253013-4723-2.

39. Mota MJ, Lopes RP, Sousa S, Gomes AM, Delgadillo I, Saraiva JA. Lactobacillus reuteri growth and fermentation under high pressure towards the production of 1,3-propanediol. Food Res Int. 2018;113:424-32. https:// doi.org/10.1016/j.foodres.2018.07.034.

40. Talarico TL, Dobrogosz WJ. Chemical characterization of an antimicrobial substance produced by Lactobacillus reuteri. Antimicrob Agents Chemother. 1989;33(5):674-9. https://doi.org/10.1128/AAC.33.5.674.

41. Santos F, Teusink B, Molenaar D, Van Heck M, Wels M, Sieuwerts S, et al. Effect of amino acid availability on vitamin B12 production in Lactobacillus reuteri. Appl Environ Microbiol. 2009;75(12):3930-6. https://doi.org/10.112 8/AEM.02487-08.

42. Santos F, Wegkamp A, De Vos WM, Smid EJ, Hugenholtz J. High-level folate production in fermented foods by the B12 producer Lactobacillus reuteri JCM1112. Appl Environ Microbiol. 2008;74(10):3291-4. https://doi.org/10.112 8/AEM.02719-07.

43. Santos F, Vera JL, Lamosa P, de Valdez GF, de Vos WM, Santos H, et al. Pseudovitamin B12 is the corrinoid produced by Lactobacillus reuteri CRL1098 under anaerobic conditions. FEBS Lett. 2007;581 (25):4865-70. https://doi.org/10.1016/j.febslet.2007.09.012.

44. Thomas CM, Saulnier DMA, Spinler JK, Hemarajata P, Gao C, Jones SE, et al. FolC2-mediated folate metabolism contributes to suppression of inflammation by probiotic Lactobacillus reuteri. Microbiologyopen. 2016; 5(5):802-18. https://doi.org/10.1002/mbo3.371.

45. Lieven C, Beber ME, Olivier BG, Bergmann FT, Babaei P, Bartell JA, et al. Memote: A community driven effort towards a standardized genome-scale metabolic model test suite. https://doi.org/10.1101/350991.

46. Lachance J-C, Lloyd CJ, Monk JM, Yang L, Sastry AV, Seif Y, et al. BOFdat: generating biomass objective functions for genome-scale metabolic models from experimental data. PLoS Comput Biol. 2019;15(4):e1006971. https://doi. org/10.1371/journal.pcbi.1006971.

47. Orth JD, Thiele I, Palsson BO. What is flux balance analysis? Nat Biotechnol. 2010;28(3):245-8. https://doi.org/10.1038/nbt.1614.

48. Waterhouse RM, Seppey M, Simao FA, Manni M, loannidis P, Klioutchnikov $G$, et al. BUSCO applications from quality assessments to gene prediction and phylogenomics. Mol Biol Evol. 2018;35(3):543-8. https://doi.org/10.1093/ molbev/ms×319.

49. Saulnier DM, Santos F, Roos S, Mistretta TA, Spinler JK, Molenaar D, et al. Exploring metabolic pathway reconstruction and genome-wide expression profiling in Lactobacillus reuteri to define functional probiotic features. PLoS One. 2011;6(4):e18783. https://doi.org/10.1371/journal.pone.0018783.

50. Nelson KE, Weinstock GM, Highlander SK, Worley KC, Creasy HH, Wortman $\mathrm{JR}$, et al. A catalog of reference genomes from the human microbiome. Science. 2010;328:994-9. https://doi.org/10.1126/science.1183605.

51. Bosma EF, Forster J, Nielsen AT. Lactobacilli and pediococci as versatile cell factories - evaluation of strain properties and genetic tools. Biotechnol Adv. 2017;35(4):419-42. https://doi.org/10.1016/j.biotechadv.2017.04.002.

52. Årsköld E, Lohmeier-Vogel E, Cao R, Roos S, Rådström P, Van Niel EWJ. Phosphoketolase pathway dominates in Lactobacillus reuteri ATCC 55730 containing dual pathways for glycolysis. J Bacteriol. 2008;190(1):206-12. https://doi.org/10.1128/JB.01227-07.

53. Spinler JK, Taweechotipatr M, Rognerud CL, Ou CN, Tumwasorn S, Versalovic J. Human-derived probiotic Lactobacillus reuteri demonstrate antimicrobial activities targeting diverse enteric bacterial pathogens. Anaerobe. 2008; 14(3):166-71. https://doi.org/10.1016/j.anaerobe.2008.02.001.

54. Sriramulu DD, Liang M, Hernandez-Romero D, Raux-Deery E, Lünsdorf $H$, Parsons JB, et al. Lactobacillus reuteri DSM 20016 produces cobalamindependent diol dehydratase in metabolosomes and metabolizes 1,2- 
propanediol by disproportionation. J Bacteriol. 2008;190(13):4559-67. https://doi.org/10.1128/JB.01535-07.

55. Walther T, François JM. Microbial production of propanol. Biotechnol Adv. 2016;34(5):984-96. https://doi.org/10.1016/j.biotechadv.2016.05.011.

56. Siebert D, Wendisch VF. Metabolic pathway engineering for production of 1,2-propanediol and 1-propanol by Corynebacterium glutamicum. Biotechnol Biofuels. 2015;8(1):91. https://doi.org/10.1186/s13068-015-0269-0.

57. Mishra SK, Malik RK, Manju G, Pandey N, Singroha G, Behare P, et al.

Characterization of a Reuterin-producing Lactobacillus reuteri BPL-36 strain isolated from human infant fecal sample. Probiotics Antimicrob Proteins. 2012:4(3):154-61. https://doi.org/10.1007/s12602-012-9103-1.

58. Thomas CM, Hong T, van Pijkeren JP, Hemarajata P, Trinh DV, Hu W, et al. Histamine derived from probiotic Lactobacillus reuteri suppresses TNF via modulation of PKA and ERK signaling. PLoS One. 2012;7(2):e31951. https:// doi.org/10.1371/journal.pone.0031951.

59. Talarico TL, Casas IA, Chung TC, Dobrogosz WJ. Production and isolation of reuterin, a growth inhibitor produced by Lactobacillus reuteri. Antimicrob Agents Chemother. 1988;32(12):1854-8. https:/doi.org/10.1128/AAC.32.12.1854.

\section{Publisher's Note}

Springer Nature remains neutral with regard to jurisdictional claims in published maps and institutional affiliations.

- fast, convenient online submission

- thorough peer review by experienced researchers in your field

- rapid publication on acceptance

- support for research data, including large and complex data types

- gold Open Access which fosters wider collaboration and increased citations

- maximum visibility for your research: over $100 \mathrm{M}$ website views per year

At $\mathrm{BMC}$, research is always in progress.

Learn more biomedcentral.com/submissions 